\title{
La reestructuración de la profesión médica
}

\section{The Restructuring of Medical Profession}

\author{
Juan IRIGOYEN \\ Universidad de Granada \\ irigoyen@ugr.es
}

Recibido: 25.10 .2010

Aprobado definitivamente: 15.11.2010

\section{RESUMEN}

Desde la década de los noventa se suceden reformas y cambios de gran alcance en los servicios sanitarios. Estos son efecto de una mutación global. El aspecto más relevante de la misma es la emergencia de un nuevo paradigma tecnoproductivo que genera una transformación organizativa radical. Su indicador más elocuente es la explosión de la gestión, portadora de un nuevo proyecto y una nueva gubernamentalidad. En las organizaciones sanitarias públicas procedentes del estado del bienestar, la reestructuración derivada de las reformas presenta dificultades debido a la incompatibilidad del nuevo modelo postfordista con algunos elementos esenciales del modelo profesional médico. Esta contradicción genera procesos organizativos caracterizados por su opacidad y complejidad. El cambio tecnológico intensivo refuerza la iniciativa de la industria biosanitaria en el complejo médico-industrial en detrimento de los médicos. La convergencia de estos cambios modifica la naturaleza del trabajo médico y erosiona su autonomía profesional, en unos términos que trascienden las categorías enunciadas por la sociología de las profesiones "proletarización” y "desprofesionalización" en un contexto anterior. La expansión del complejo médicoindustrial bajo la hegemonía de las industrias se sustenta en el supuesto de crecimiento permanente y genera dudas sobre su sostenibilidad económica y social. La institución de la medicina, movilizada y orientada por innovación tecnológica y transformada en un campo de producción y consumo intensivo, genera una cosmovisión y un campo de visibilidad muy descentrado con respecto a los complejos problemas de salud colectiva, que también en las sociedades del presente se derivan de problemas sociales y desigualdades sociales.

Palabras Clave: Reformas sanitarias, gestión sanitaria, profesión médica, neoliberalismo, gubernamentalidad, desprofesionalización, organizaciones sanitarias.

\begin{abstract}
Since 1990s, healthcare services have suffered far-reaching changes and reforms that have arisen from a global mutation. The most outstanding aspect of this global mutation is the emergence of a new technoproductive model that produces a drastic organizational transformation. The most remarkable indicator is the management explosion that generates a new project and a new governmentality. The restructuring derived from the reforms mentioned above poses difficulties to the public healthcare organizations, emerged from the welfare state, due to the incompatibility between the new Post-Fordist model and some basic elements of the medical professional model. This conflict generates organizational processes
\end{abstract}


characterized by opacity and complexity. The intensive technological change reinforces the leadership of the biomedical industry inside the medical-industrial complex to the detriment of the physicians. The confluence of these changes transforms the nature of medical practice and erodes his professional autonomy in a way that transcends the categories previously stated by the sociology of the professions, "proletarianization" and "deprofessionalisation". The increase of the medical-industrial complex under the hegemony of the industries is supported on an alleged constant growth and generates doubts on its social and economic sustainability. The medical institution, powered and guided by the technological innovation and transformed into an intensive consumption and production field, generates a very decentralized scope and worldview with respect to the complex collective health problems that also derive from social inequality and social problems in current societies.

KEYWORDs: Sanitary reforms, health management, medical profession, neoliberalism, governmentality, deprofessionalization, health care organizations.

\section{SUMARIO}

1. La mutación global y las reformas sanitarias del presente. 2. El modelo postfordista de producción de servicios sanitarios. 3. Las nuevas organizaciones sanitarias: el advenimiento de la gestión y la nueva gubernamentalidad. 4. La profesión médica en la reestructuración de los servicios sanitarios. 


\section{LA MUTACIÓN GLOBAL Y LAS REFORMAS SANITARIAS DEL PRESENTE}

Desde los años ochenta se produce una secuencia de cambios de gran profundidad en el campo de la salud y de los servicios sanitarios. En España, el "Informe Abril" en 1991 abre este proceso de transformación del sistema sanitario. Los cambios que se realizan afectan a todas las esferas del mismo y generan un notable desconcierto entre los profesionales. Se trata de cambios exógenos que se introducen desde el entorno. De esta transformación resultan nuevas finalidades, discursos, prácticas asistenciales y dispositivos organizativos. La puesta en marcha de esta espiral de cambios, no se promueve por la iniciativa de la profesión médica, sino por la de las autoridades sanitarias y los gestores. Los profesionales los siguen adaptándose a las nuevas situaciones y tratando de conservar sus posiciones y prerrogativas

Los cambios registrados en el campo sanitario se encuentran conectados con transformaciones que se producen en ámbitos más globales. Pero existe una notable confusión acerca de la naturaleza y la causalidad de los cambios. Se carece de un marco teórico que tenga la capacidad de ordenar y priorizar los cambios múltiples que son percibidos de forma dispersa. El declive relativo de las ciencias sociales frente a la emergencia de un conjunto de discursos y saberes procedentes del campo de la tecnología, la empresa y la gestión, propicia la generalización de conceptualizaciones manifiestamente triviales en relación a la complejidad social. Las distintas versiones de la sociedad de la información y el conocimiento consolidan la confusión existente, acrecentada por un torrente de novedades que se presentan desbordando los supuestos de estas interpretaciones.

Las teorías de la sociedad de la información y el conocimiento entienden los cambios sociales derivados del cambio tecnológico. A partir de este núcleo se desencadenan procesos de cambio social que acompañan a las transformaciones tecnológicas. De este modo, otras dimensiones son subordinadas o excluidas de su campo de análisis o problematización. Así se contribuye a la invisibilización de áreas relevantes de las realidades sociales. Las categorías, los lenguajes y las codificaciones que articulan estos discursos, construyen un marco cognitivo desde el que se interpretan los acontecimientos con marcado optimismo. Su campo de visibilidad les permite ignorar hechos sociales externos a su discurso. Estas teorías son las que predominan en el campo sanitario introducidas desde la hegemonía de la gestión.

Desde las ciencias sociales existen distintas interpretaciones de las mutaciones en curso, dando lugar a un cuadro relativamente complejo, en el que las diferencias se pueden clasificar según la selección de la dimensión central del cambio que opera en cada teoría. Las conceptualizaciones procedentes de las ciencias sociales son más consistentes y pluridimensionales que las propuestas de la sociedad de la información/conocimiento apuntadas. Varían en su grado de complejidad y su área de problematización. Con la excepción de la propuesta de sociedad postindustrial de Bell, de la que algunas versiones de las teorías de información/conocimiento toman su esquema básico evolucionista y unilineal, las interpretaciones de la mutación son más elaboradas y están dotadas de un dispositivo conceptual más sólido. La sociedad-red, la modernización reflexiva, las teorizaciones acerca de la sociedad de riesgo, el capitalismo desorganizado, los diferentes análisis en torno a la postmodernidad, las distintas versiones postfordistas y las teorías de última generación, tales como el capitalismo cognitivo, la transmodernidad, las teorias posdecoloniales o decoloniales y las sociedades postdisciplinarias o de control.

El cambio global, exógeno al sistema sanitario y que lo reconfigura en el presente, es el efecto de una mutación de gran alcance que involucra a la sociedad en su conjunto. Se trata en su origen de una gran crisis del capitalismo en los años setenta, que se manifiesta como agotamiento del sistema productivo del que resulta el proceso de desindustrialización de los años ochenta. El desplome del antiguo sistema productivo es simultáneo al nacimiento de un proceso de reindustrialización sobre nuevas bases tecnológicas y organizativas, del que se deriva una reestructuración global en todas las esferas. Una de las dimensiones fundamentales de la transformación radica en la crisis de las instituciones reguladoras del orden social anterior, que 
siguen funcionando en el nuevo contexto, siendo requeridas para su transformación. Podemos designar esta mutación como un cambio esencial en la economía, la producción, el estado, las instituciones, la norma de consumo, la cultura, la estratificación social, la individuación y las subjetividades.

La mutación global tiene como consecuencia la emergencia de una nueva sociedad que se asienta sobre bases tecnológicas nuevas, en tipos organizativos muy diferenciados de las organizaciones complejas del fordismo-keynesianismo y en un sistema de relaciones de poder inédito. La nueva empresa representa emblemáticamente esta sociedad que irrumpe generando instituciones muy diferenciadas de las convencionales: los nuevos media, la constelación de la 2.0 y las redes sociales, la publicidad, el marketing, la gestión, las organizaciones de producción de conocimiento (fundaciones, think tanks) y las diversas categorías de organizaciones y expertos que desempeñan funciones relevantes en el nuevo orden. Esta sociedad emergente es muy dinámica. Genera una gran energía y creatividad adquiriendo un alto grado de legitimidad.

Junto a la sociedad emergente se configura otra sociedad diferente, aquella formada por las grandes instituciones, organizaciones y segmentos de la economía florecientes en la anterior sociedad industrial fordista-keynesiana, cuyo origen se ubica en contextos sociohistóricos muy diferentes, que han configurado su modelo institucional en coherencia con las condiciones sociales existentes en su fundación. En el presente no encajan bien con la naciente sociedad. Todas estas esferas sociales son reestructuradas para adaptarlas a los nuevos requerimientos y coherencias globales, así como a los intereses hegemónicos que impulsan los cambios. De ahí las sucesivas reformas de la enseñanza, la universidad, el sistema sanitario, los servicios sociales y las organizaciones que articulan el estado. La complejidad de las distintas realidades organizacionales de las instituciones a reconvertir, determina que las reformas se encuentren con grandes obstáculos. Así se explica la continuidad de la espiral de reformas sin fin. Junto a estas dos sociedades se encuentran otras en declive o en trance de desaparición.

Una cuestión fundamental, eludida en muchas teorías, es la política. La crisis de los años setenta y la nueva reindustrialización, implica la ruptura de los equilibrios de intereses existentes en las sociedades industriales hasta los años setenta. Las nuevas bases tecnológicas y organizativas proporcionan ventajas sustanciales a los grandes intereses de las empresas y corporaciones. El debilitamiento de los trabajadores, de los sindicatos y de distintos segmentos sociales fragmentados es patente. Pero es en el plano del conocimiento donde la desigualdad es mayor. La mutación global determina el desplome de las teorías y saberes convencionales que han sustentado a la izquierda política y social. Esta se encuentra desbordada y perpleja ante las nuevas realidades. El vacío que se genera lo rellena el neoliberalismo. Este es un proyecto congruente con los grandes intereses económicos que aprovecha las oportunidades que le proporciona la reestructuración y ejerce su hegemonía decididamente. El punto fuerte del neoliberalismo es su gran versatilidad y flexibilidad. Puede estar presente en distintos contextos operando simultáneamente en condiciones muy diferenciadas.

El neoliberalismo se estructura e interviene más allá del espacio político convencional. Configura un nuevo espacio público mediante las reformas gerencialistas de la administración, que extienden la gestión a todos los sistemas organizativos. Desde la gestión se genera una red de organizaciones nuevas: fundaciones, think tank, agencias de evaluación, empresas y organismos de servicios especializados, de innovación y otras, que configuran las fuerzas estructurantes transversales que impulsan las transformación en la dirección de su proyecto. Un requisito esencial para el avance del proyecto neoliberal es debilitar los círculos sociales, los poderes autónomos y las rutinas que frenan la expansión de los mercados. La burocracia y las profesiones son las formas procedentes de la sociedad industrial convencional que es necesario transformar para recomponer las coherencias con el nuevo conjunto. La reestructuración significa un desplazamiento de las élites sociales características de la época anterior, y la emergencia de nuevas élites congruentes con el proyecto neoliberal.

Los cambios continuos y dispersos procedentes del entorno en los últimos años, que son percibidos como extraños desde el interior de 
los servicios sanitarios, resultan ser parte de una propuesta integrada coherente con la sociedad hegemónica naciente y el proyecto que la inspira. Las reformas sanitarias del presente significan la importación del modelo de la nueva empresa resultante de la reindustrialización al campo sanitario, así como la reconfiguración del estado, que se entiende desde la función esencial de servir al crecimiento económico estimulando el mercado. Este es su verdadero significado. Pero como no es posible transplantar la totalidad del modelo neoliberal a las organizaciones sanitarias configuradas en el estado del bienestar, la estrategia adoptada consiste en la introducción gradual de elementos y dosis de mercado en la organización burocrática y profesional. La complejidad de los procesos que se abren es patente.

Se modifican los lenguajes y el conocimiento, los profesionales devienen en proveedores y los pacientes en clientes, pero se confirman las dificultades de reformar las prácticas médicas y los servicios asistenciales. Las viejas jerarquías de la organización burocrática y profesional consolidan sus posiciones y revierten los significados de las reformas. Se configura una organización que resulta de la hibridación entre elementos de los viejos y nuevos modelos.

Las reformas operan sobre un sector que puede ser considerado como un campo social. Después de Bourdieu existe consenso en reconocer que en un campo existe un conjunto de posiciones, relaciones de fuerza, monopolios, jerarquías, procesos de redistribución, estrategias, intereses y beneficios. Sobre los equilibrios imperantes en el campo sanitario de los años noventa, la reforma significa el aumento de la densidad y complejidad del campo al intervenir actores nuevos. Los malestares inespecíficos generados por las reformas sanitarias tienen su explicación en el incremento de la densidad del campo, que implica la modificación de los procesos de interacción operativos con anterioridad.

La sociología de las profesiones ha conceptualizado las sucesivas transformaciones de las mismas, que han modificado su naturaleza adaptándose a los cambios producidos en los diferentes contextos en las que se inscriben. Se trata de un campo evolutivo de gran complejidad teórica, en el que convergen distintas líneas de desarrollo y que realiza importantes apor- taciones a la sociología. En lo que se refiere a la profesión médica, se han formulado distintas conceptualizaciones desde Parsons hasta el presente, constituyendo uno de los núcleos de la sociología de la salud. En la evolución de todas las teorías acerca de la profesión médica, se puede apuntar la convergencia en la idea de declive profesional. El ejercicio en organizaciones complejas y la evolución del entorno social, que modifica la posición de los pacientes, erosionan el monopolio profesional y la legitimidad de la profesión. Las teorías de los años cincuenta, que enfatizan los valores y las funciones de la profesión médica, se van modificando introduciendo sucesivos elementos críticos.

La hipótesis del presente trabajo se ubica en esta perspectiva de declive de la profesión médica. Pero las transformaciones del contexto socio-histórico son de tal magnitud, que hacen necesario reconceptualizar el presente de la profesión. La cuestión fundamental no estriba tanto en el declive profesional, comparado con situaciones anteriores, sino en un proceso de reprofesionalización para ser adaptada a las exigencias de los poderosos intereses económicos que controlan el campo de la asistencia sanitaria, los imperativos de los tipos organizativos nacidos de la transformación tecnológica y de la reconfiguración del estado. La medicina como institución es reconstituída para ser integrada en el orden emergente neoliberal. Un elemento primordial de la reprofesionalización es el comienzo de un proceso de fragmentación y jerarquización interna, cuyo resultado es el contraste entre la opulencia de las élites profesionales beneficiarias de la expansión terapeútica y los sectores perjudicados por la reestructuración del sistema público, que los convierte en segmentos profesionales cuyo ejercicio profesional se encuentra severamente limitado por la restricción de recursos diagnósticos y terapéuticos.

La reestructuración de los servicios sanitarios, así como de la profesión médica se realiza mediante el desarrollo de la gestión. Esta es portadora de la nueva gubernamentalidad del presente. La gubernamentalidad es un concepto formulado por Foucault. Desde esta perspectiva se entiende la realidad de las organizaciones sanitarias donde ejerce la profesión médica, como un espacio de gobierno. La gubernamentalidad implica racionalidades de gobierno, tec- 
nologías de gobierno y métodos de conducción. La gestión impulsa la reestructuración a partir de una mutación de las reglas de producción de poder y del modo de gobierno que reduce progresivamente la autonomía y las bases de poder de los profesionales. La nueva gubernamentalidad articula todo el proceso de reconversión de los servicios sanitarios y de las profesiones.

En la primera parte del trabajo se analizan los cambios económicos, tecnológicos, organizativos y culturales que modifican la producción de los servicios sanitarios. Se subraya la reconfiguración de todo el campo determinado por el incremento de poder de la industria. En la segunda parte se explican los procesos de redistribución de poder en las organizaciones sanitarias como efecto de la progresiva aplicación de la nueva gubernamentalidad introducida desde la inteligencia rectora del sistema global. En la última, se exploran los principales dilemas de la nueva medicina gerenciada en relación con los problemas de salud pública característicos del presente. La complejidad de éstos no se corresponde con los sentidos de la reestructuración de la profesión promovida por los agentes y estructuras de poder globales.

\section{EL MODELO POSTFORDISTA DE PRODUCCIÓN DE SERVICIOS SANITARIOS}

La mutación iniciada en los años ochenta modifica sustancialmente los dispositivos técnicos y organizativos de la producción. De su desarrollo nace una empresa completamente nueva, muy diferenciada de las empresas que operan en el fordismo maduro. En el contexto postfordista concurren dos factores esenciales: el cambio tecnológico continuo y un mercado expansivo y movilizado. Ambos factores imponen unos requerimientos rigurosos y configuran la nueva organización. Esta tiene que adaptarse a las dinámicas producidas en ambas esferas.

El cambio tecnológico resultante de la ruptura tecnológica de los años ochenta se configura como un factor determinante (Ominami, 1986). Las nuevas tecnologías se encuentran en una fase de desarrollo en la que generan prestaciones que permiten añadir mejoras a los objetos o servicios producidos, o bien inventarlos com- pletamente nuevos. Todo ello en ciclos temporales cortos, resultando un alto grado de renovación y aceleración. El sistema tecnológico se articula en una red heterogénea y global de instituciones y organizaciones de investigación. Las empresas tienen que tener la capacidad de conectar y explorar las posibilidades que ofrece este dispositivo tecnológico.

Los mercados son muy dinámicos. Las sociedades contemporáneas se encuentran muy segmentadas. Distintos grupos realizan valoraciones, generan aspiraciones y desencadenan procesos de comunicación. Así se genera un entramado entre segmentos de consumidores, medios de comunicación y empresas, que conforma un espacio donde tienen lugar distintos procesos de invención, comunicación e influencia. La empresa interactúa en este espacio dinámico y opaco. La comunicación se transforma en el vector más importante de la acción empresarial. Los sistemas de interacción con los clientes y la gestión de valores intangibles, se conforman como actividades que trascienden a la misma producción material y constituyen la principal fuerza estratégica (Costa, 1999). La detección de insatisfacciones o la interpretación de las señales débiles realizadas por los clientes, adquieren una importancia primordial.

La nueva empresa vive entre estos dos mundos dinámicos, lo que implica la necesidad de poseer y desarrollar activos de conocimiento para asegurar su competividad. Desde esta perspectiva se puede comprender la reconversión organizativa. Se trata de constituir un tejido vivo que favorezca la interacción y comunicación interna, que elimine las barreras departamentales y permita la circulación de los flujos de conocimiento. La organización, gestionando adecuadamente sus procesos esenciales, hace posible la integración de todas las fases del ciclo de producción. Una cuestión relevante es el estímulo de la creatividad, puesto que en las condiciones de un entorno tecnológico y de mercado que se transforma a gran velocidad, la innovación es un requisito fundamental para mantener la competividad.

La empresa postfordista produce conocimiento. Este es el principal activo y la competencia esencial de la misma. Ya no se trata de producir bienes que satisfagan las necesidades sino de producir conocimientos y tecnologías 
que se hagan cargo de la satisfacción de las necesidades que se generan mediante estimulación e interacción. El output final está formado por los conceptos de producto obtenidos a partir del saber. La explosión del conocimiento ha dado lugar a distintas valoraciones contrapuestas. De un lado, Drucker (1993) sintetiza la visión aproblemática de la producción y gestión del conocimiento. La economía del conocimiento los produce y distribuye en una trama de organizaciones reguladas por el management, entendido como una tecnología científica de dirección. Los participantes en el proceso son los trabajadores del saber, que se definen en términos de un grupo privilegiado por la naturaleza de la función que desempeñan.

Otras interpretaciones problematizan los procesos de producción de conocimiento (Ayestarán, 2007) (Moulier-Boutang, 2007) en un sistema definido como capitalismo cognitivo. Desde estas perspectivas se muestran las tensiones generadas por la apropiación y privatización del conocimiento por parte de las empresas. Este es un producto obtenido mediante la cooperación y la inteligencia colectiva de la producción inmaterial (Lazzarato y Negri, 2001). En las conceptualizaciones del capitalismo cognitivo, los trabajadores inmateriales, brainworkers, son definidos en términos problemáticos. Sus aportaciones trascienden el tiempo de trabajo y los perfiles profesionales. Sus itinerarios profesionales muestran los procesos de intermitencia, precarización y vulnerabilidad. El trabajo se funda en el saber común, en la cooperación y en las capacidades expresivas (Berardi, 2003) y se extiende a toda la vida, no siendo reductible a unidades de tiempo. El valor del conocimiento radica paradójicamente en su privatización, en su acceso restringido, en la limitación temporal de su difusión. El balance de la relación entre los brainworkers y las empresas es de intercambio desigual, siendo las aportaciones de éstos escasamente proporcionales a las recompensas recibidas.

Desde estas perspectivas se suscita otra cuestión que tiene un impacto importante en el campo de la salud. El concepto conocimiento no sólo implica un saber tecnológico y material. También es un saber social : "conocimiento es un saber social sedimentado o manifiesto, que vislumbramos en las relaciones sociales, expre- siones, códigos, costumbres, formas de comunicación, esquemas de interpretación, valores, etc, que representan también para las corporaciones un valioso filón de materia prima que hay que transformar y reconducir a la esfera de lo funcional" (Quintana, 2009, 360). La producción en el postfordismo requiere penetrar en la vida de los consumidores más allá de los targets convencionales. Se trata de poner toda la vida a trabajar. Desde estas coordenadas se entiende la centralidad de la comunicación y su fusión con la producción (Marazzi, 2003). Se construye un dispositivo de captura de significaciones y un aparato de observación y análisis que va más allá del marketing convencional unidireccional. La interacción con los consumidores implica la transformación de sus cogniciones en demanda. Se constituye un proceso de fabricación de necesidades mediante la estimulación permanente de los satisfactores.

Las nuevas empresas en el ciclo de producción inmaterial (Lazzarato, 2009) requieren de un modo de dirección del conjunto muy diferente a la antigua dirección jerárquica. Nace la gerencia, la gestión molecular cuya principal función es producir organización. Aquí radica una de las claves de la época. Esta es una nueva institución que desempeña una función fundamental más allá de la dirección convencional. La gestión todavía se encuentra en su infancia. Ahora no se trata de dirigir un conjunto de personas que venden su tiempo de trabajo, sino de maximizar las calificaciones útiles del capital humano. Los brainworkers tienen que estar dotados de variadas cualificaciones y desarrollar interacciones, comunicaciones y producciones que trascienden cualquier tiempo de trabajo definido convencionalmente en horarios. La gestión implica la estimulación de los agentes desde un nuevo poder dotado de sensibilidad localizada, capaz de producir sinergias.

La producción de los servicios sanitarios se realiza en las organizaciones sanitarias. La profesión médica desempeña un papel fundamental en el proceso. El trabajo médico consiste básicamente en la realización de diagnósticos y tratamientos a pacientes individuales. Estas actividades se realizan apoyadas en medios técnicos producidos industrialmente. Una parte del trabajo médico se apoya en el manejo de distintas tecnologías. Los laboratorios, la radiología y 
otros medios, desempeñan un papel fundamental en los procesos diagnósticos, así como los medicamentos, la cirugía y otros tratamientos constituyen las distintas alternativas terapéuticas. El progreso de la biomedicina se ha apoyado en el incremento de medios diagnósticos y terapéuticos. Estos han constituido una poderosa industria que transfiere sus resultados al campo clínico.

La reestructuración global iniciada en los ochenta se funda en la ruptura tecnológica y en la aparición de nuevas tecnologías que impulsan el crecimiento económico y la potencialidad de la industria. En el sector biomédico la expansión tecnológica se manifiesta en el desarrollo de las biotecnologías y su interacción con las TIC y otras tecnologías fundamentales. Los resultados son relevantes en los últimos veinte años en numerosos campos y aplicaciones. Este salto tecnológico permite disponer de nuevos medicamentos y medios diagnósticos y terapéuticos. La industria biomédica se desarrolla extraordinariamente y se configura como una de las que más contribuye al desarrollo de la nueva economía (Acebillo y Artells, 2004).

En los años setenta, Arnold Relman, en un discurso a la Massachussets Medical Society, utilizó un concepto refiriéndose a la expansión de las industrias de la salud: Complejo MédicoIndustrial. Su intención era construir una analogía con el complejo militar industrial desarrollado en los años anteriores. El atributo fundamental de este complejo era su influencia y poder político y económico. El complejo médico-industrial es un dispositivo compuesto por las industrias de tecnología médica, la industria farmacéutica, las empresas de atención médica, las organizaciones sanitarias y las profesiones sanitarias (Sobrino, 2010). El complejo integra la base científico-técnica que radica en las universidades e instituciones de investigación, las industrias sanitarias y la investigación clínica.

Siempre ha existido una estrecha relación entre la industria y la atención médica. Pero la ruptura tecnológica de los ochenta modifica sustantivamente esas relaciones. Ahora la industria ha incrementado su poder basado en su potencialidad tecnológica. El incremento de su producción y actividad la ha convertido en uno de los sectores productivos industriales que más contribuye al crecimiento económico general.
La expansión de la sanidad radica en la potencialidad de sus mercados, su alta productividad y competitividad, así como sus resultados económicos. La industria biomédica genera incrementos en inversiones, consumos, empleos cualificados, demandas y expectativas crecientes.

La expansión de la industria biomédica le proporciona una preponderancia tanto en la investigación clínica como en el campo asistencial, en el que las innovaciones se derivan principalmente de la industria. Los ensayos clínicos son el espacio de encuentro y cooperación entre la industria y los profesionales de la asistencia. En los últimos años se han suscitado numerosas críticas respecto a éstos. La falta de rigor en los diseños, los problemas con las muestras o la evaluación de los resultados y su comunicación son los más importantes. Pero el problema de fondo radica en que es la industria quien selecciona a los profesionales que colaboran en los ensayos clínicos. Su posición de fuerza se basa en la financiación de los mismos, derivando ingresos económicos a los profesionales y centros, y en el control de los procesos de producción de conocimiento e innovación. De este modo la industria toma la iniciativa y construye un entramado de relaciones de profesionales, agencias, fundaciones e instituciones, en las que su preponderancia es incuestionable. Desde esa posición desarrolla estrategias de fidelización con su red de colaboradores, generando relaciones clientelares (Sobrino, 2010).

La comunicación es un vector esencial en la producción postfordista. La industria biomédica desarrolla una comunicación comercial efectiva en el formato publicitario de la época. Las necesidades son seleccionadas y reconstituidas desde la industria según sus objetivos. Los profesionales colaboradores se ven reducidos a un papel secundario de acompañantes en la promoción de los productos. Los medicamentos protagonizan la expansión terapéutica de las últimas décadas. De este modo, se constituye un modelo médico basado en la seducción comercial que es importado desde la industria (Borracci, 2001, 2006).

El problema de la industria farmacéutica estriba en su necesidad de innovación permanente. Muchos de los productos que promociona como si fueran nuevos, son versiones mejoradas de productos ya existentes. Todos los elementos reseñados anteriormente de la producción pos- 
tfordista se encuentran presentes en la misma. Los gastos de I+D son elevados, los de fabricación son bajos y los de promoción representan la mayor parte de los costes. Con una capacidad limitada de innovación, tiene que desarrollar sofisticadas estrategias de protección jurídica de sus productos. En las coordenadas del capitalismo vigente las inversiones se movilizan hacia los sectores que proporcionen una ratio adecuada beneficios/capital invertido (Pignarre, 2005).

La situación de la industria farmacéutica implica su movilización intensiva. Se constituye como un lobby poderoso que construye un dispositivo de presión e influencia en el estado, los servicios sanitarios, los profesionales y la opinión pública. La industria farmacéutica ilustra el modo de operar de las reformas neoliberales que proceden al debilitamiento del estado mediante la cooptación de sus recursos humanos. Estas prácticas denominadas de "puerta giratoria" (the revolving door), consisten en la contratación de directivos y funcionarios relevantes de los sistemas públicos estatales. La industria también penetra en la intelligentsia de la profesión médica por distintas vías como empresas consultoras $\mathrm{y}$ de investigación (Angell, 2004).

La relación entre la industria biomédica y su entramado de colaboradores proporciona gradualmente un mayor poder de definición de las necesidades y de los problemas de salud en detrimento de la profesión médica. Los proyectos se deciden en función de su potencialidad comercial. De este modo los enfermos son transformados en consumidores segmentados y las prioridades se concentran en los problemas de salud que afectan a los segmentos con mayor capacidad de compra. La profesión médica debilita su hegemonía en la definición de los problemas de salud desplazada por el aparato productivo industrial. La explosión de la asistencia médica comercializada bajo el control de la industria, produce una nueva medicalización en la que los médicos colaboran en las iniciativas de la industria dirigida a los nichos de mercado. Algunos problemas relacionados con el bienestar físico son transformados en enfermedades y se modifican criterios diagnósticos de modo que se incremente la demanda. De esta forma se configuran los inventores de enfermedades (Blech, 2005).

Una cuestión primordial es la del valor. Si este se puede definir como la diferencia entre el precio del producto y los insumos necesarios para su obtención, la realidad es que el factor determinante de su valor es su consumo. La necesidad de aumentar tanto el consumo como la disposición a pagar, constituye el núcleo de la estrategia de la industria. En coherencia con esta definición, los énfasis en conseguir colaboradores entre los profesionales así como en la comunicación, deviene en una cuestión fundamental. El efecto perverso de la industria es la construcción de su propia demanda para los proyectos en los que el costebeneficio sea favorable. En este proceso algunas categorías de pacientes con enfermedades con déficit terapéutico son desplazados de los proyectos de investigación y de producción, por razones principalmente económicas (Jara, 2007). Las prioridades de la industria y las de salud pública se encuentran disociadas. El modo de constituir nuevos segmentos de mercado radica en la realización del marketing del miedo (Jara, 2009).

La complejidad de la producción de servicios sanitarios es patente. La industria, el dispositivo de investigación, las autoridades comprometidas prioritariamente con el crecimiento económico, los inversores, las instituciones sanitarias, las profesiones y las múltiples categorías de enfermos. Los médicos se encuentran atrapados entre la industria que presiona para la expansión del consumo y las autoridades que imponen criterios eficientistas restrictivos. El ejercicio profesional es un espacio en el que se registran tensiones y dilemas difíciles de resolver. Pero la preponderancia del complejo industrial se manifiesta en una hegemonía en la definición de las prácticas asistenciales. Los sistemas públicos adoptan modelos inspirados en el mercado, en la que el producto se entiende como un servicio complejo y el destinatario como un cliente. La asistencia sanitaria pública, referenciada en el universalismo propio de las instituciones del fordismo-keynesianismo, es reformada gradualmente para configurarse en un modelo postfordista fundado en la estratificación y las diferencias.

La producción de servicios sanitarios representa todos los elementos de las economías del conocimiento postfordistas apuntadas con anterioridad. Los médicos son trabajadores inmateriales que toman decisiones clínicas sobre la base de un conocimiento producido cooperativamente por un dispositivo heterogéneo de inteligencia colectiva y comunicación. Una parte de sus decisiones 
terminan en consumos de productos constituidos en mercancías. La reestructuración global modifica el alto grado de autonomía profesional existente hasta los años ochenta. El poderoso complejo de industrias de medios diagnósticos, farmacéutica, de biología, de la alimentación, de compañías aseguradoras, empresas consultoras y de recursos humanos, se hace presente en el campo de la asistencia con gran intensidad. Este complejo financia publicaciones profesionales, actividades de formación médica continuada, congresos médicos, comités de expertos para problemas de salud, sociedades científicas y asociaciones de enfermos. En estas nuevas condiciones nos podemos interrogar acerca del campo de fuerzas en el que se define la autonomía profesional.

La cuestión más importante en la producción postfordista de los servicios sanitarios radica en la estimulación de las necesidades característica de la producción inmaterial. La necesidad imperativa de crecer los mercados, genera un consumidor equivalente al de los mercados de servicios. Este siempre se encuentra en estado de alerta para maximizar su bienestar mediante la movilización inmediata de todo el poder terapéutico disponible. La publicidad de productos farmacéuticos se constituye en un emblema del capitalismo cognitivo, capturando las significaciones y prácticas de los consumidores que son transformadas en elemento de seducción. Los efectos en la asistencia médica de esta cultura de consumo desbordan el clásico concepto de efectos perversos formulado por Boudon. Crean un tipo de consumidor que tiende a eludir su responsabilidad transfiriéndola a los profesionales. La tesis de tentación de la inocencia (Bruckner, 1996), ilustra este problema. Los problemas que requieren asistencia sanitaria siguen estando vinculados a la compleja relación entre las condiciones de vida y el comportamiento individual y social.

\section{LAS NUEVAS ORGANIZACIONES SANITARIAS: EL ADVENIMIENTO DE LA GESTIÓN Y LA NUEVA GUBERNAMENTALIDAD}

La mutación global que se inicia en los ochenta determina una profunda reestructuración del estado. En las controversias acerca de su naturaleza se mantiene un área oculta mani- fiesta. El objetivo de las reformas radica en adaptarlo a la lógica del conjunto, a los requerimientos del sistema productivo emergente y al proyecto hegemónico neoliberal. Se trata de transformar el antiguo estado del bienestar para convertirlo en un estado técnico y profesional, eficiente, que actúe con una lógica económica eficaz subordinándose a los equilibrios macroeconómicos, que contribuya a la diversificación de la oferta y a una privatización de las opciones de la demanda. Este estado debe abrir espacios para que el mercado actúe. Su objetivo principal es contribuir a la potenciación de la competitividad económica y el crecimiento.

Las organizaciones de producción de servicios públicas, encuadradas en los estados, importan la racionalidad económica y el costobeneficio se transforma en el principio rector de las organizaciones públicas. El nuevo estado proyectado como relacional, que tiene que subsistir compitiendo y cooperando con las organizaciones del mercado, asume como referencia el modelo empresarial. La nueva empresa postfordista se define a partir de la gestión. En la propuesta de la Nueva Gestión Pública están presentes un conjunto de elementos que son unificados y catalizados por la gestión. Esta es la primera fuerza para la reconversión de todas las organizaciones, un elemento trasversal que asegura los vínculos con el entorno (Hassard y Parker, 1997).

La reforma del estado en los servicios sanitarios presenta grandes dificultades. El modelo de empresa postfordista no encaja con las condiciones de una organización bajo el dominio profesional. La complejidad del sistema sanitario público estriba en la dificultad de la medición del producto, el poder profesional en la toma de decisiones clínicas y el limitado poder de los gerentes. La reducción de los recursos disponibles y el aumento de costes, determinado principalmente por la tecnología, plantea un problema que no se puede resolver sin la incorporación de los médicos a la gestión de modo que asuman criterios eficientistas. El problema se agudiza en tanto que se pone fin a una etapa de expansión de los recursos sanitarios que queda inscrita tanto en los imaginarios profesionales como en los de la población.

Las organizaciones médicas hasta los años ochenta se han regulado mediante la autoges- 
tión. La dirección se limita a la administración de los procesos desempeñada por un clínico que considera su etapa en la dirección como un paréntesis. La importación del modelo de empresa en la reestructuración, implica la aparición de la gestión, la definición del producto como un servicio que tiene que homologarse con el sistema de servicios personales imperante, y la definición de los pacientes como clientes. La atención médica es un sistema de supuestos y sentidos que es sustancialmente alterado con la irrupción de modelos de mercado portadores de sus propios sentidos consumeristas (Irigoyen 2007). Los comienzos de la reforma registran un conflicto, no siempre manifiesto, cuyos contenidos expresan un shock cultural.

En un proceso productivo tan intensivo, la presencia de lo profesional interfiere el modelo e inmoviliza a la gerencia, debido a la rigidez de las regulaciones laborales y a las áreas que no son negociables en tanto que se corresponden a regulaciones de las profesiones. Los límites a la eficiencia, contención del gasto y determinación de la calidad son patentes. Uno de los instrumentos más efectivos del modelo de mercado, los incentivos, también tiene la eficacia limitada debido a la sólida fidelidad corporativa. La flexibilización de los procesos o formación de equipos encuentran obstáculos difíciles de remover. La gestión de los recursos humanos tropieza con el control de los profesionales de la formación, la contratación y la definición de las prácticas asistenciales. Los instrumentos clave en la empresa, como la gestión de las competencias o la promoción profesional, también se encuentran constreñidos en organizaciones profesionales.

El elemento fundamental para la reconversión de la organización profesional es la gestión. Esta no es sólo una forma de dirección, sino una institución nacida en el presente, constituida como parte esencial del proyecto económicopolítico en curso, representante de la estructura central, el mercado, e investida de legitimidad para transformar las viejas organizaciones y generar condiciones propicias para el desarrollo de los mercados (Parker, 2002).La gestión se presenta como modelo único y reemplaza gradualmente la autogestión de los profesionales. $\mathrm{Su}$ discurso se articula en torno a un precepto fundamental: se define como un elemento de progreso respecto al pasado burocrático, y cualquier cuestionamiento es interpretado en término de atraso cultural. Los servicios sanitarios son el escenario donde se interseccionan dos proyectos, el de los gerentes y el de los profesionales.

Existen varias interpretaciones críticas con la gestión. Entre las mismas destacan los autores anglofoucaultianos que desarrollan el concepto de gubernamentalidad. Esta se entiende como un modo de gobierno formado por racionalidades políticas y tecnologías de gobierno. Foucault la define como "un conjunto de" instituciones, procedimientos, análisis, reflexiones, cálculos, tácticas, y controles reguladores diseminados "que permiten ejercer el poder" (Foucault, 1981, p.25). La gubernamentalidad se define en relación a contextos históricos específicos. En el presente emerge una gubernamentalidad neoliberal (Rose, 1997, 2007)). Esta se entiende como un modo de gestionar los procesos sociales de modo que se aproveche la energía que aporten los propios gobernados. Se trata de programar los entornos de los individuos, de modo que ellos mismos se autorresponsabilicen y decidan entre las opciones disponibles. Las tecnologías de gobierno incentivan las conductas basadas en el cálculo, la previsión del futuro y el autocontrol. Los individuos son estimulados a ser activos y desarrollar iniciativas. Se trata de encontrar la intersección entre la conducta personal y los objetivos de gobierno. Esta forma de poder fomenta la persuasión y la seducción minimizando la coacción explícita.

La nueva gubernamentalidad se desarrolla en todas las esferas y en las organizaciones en particular. La gestión es representativa del nuevo modo de conducción (du Gay, 2000). Su objetivo es hacer funcionar la red de proyectos y maximizar la aportación de las personas y equipos. El modelo de la tecnología de la gestión se corresponde con las condiciones del capitalismo cognitivo y con la gestión de los brainworkers. No obstante, continúan existiendo muchas prácticas de gestión que remiten al sistema de dirección taylorista. Pero la gubernamentalidad emergente es la definida como neoliberal (Rose y Miller, 1992). Esta es una forma de conducir los procesos mediante la instrumentación de la relación. La gubernamentalidad neoliberal es aplicada en la reestructuración del estado y de 
los servicios sanitarios, configurándose como un elemento constituyente de la gestión ((McKinlay y Starkey, 1998).

Una interpretación de la gestión en organizaciones profesionales es la de Ball (1993). Este la entiende siguiendo a Foucault como una tecnología moral. Es una tecnología individualizadora que genera un campo permanente de visibilidad para cada sujeto. Se producen informes y documentos que terminan construyendo a cada uno como un sujeto visible, descriptible y comparable. De este modo se configura como un instrumento de control y disciplinamiento, aunque el objetivo es la autorresponsabilización. Así, mediante la evaluación, se construye un poder operativo sobre los evaluados que los excluye de facto de las decisiones generales. La gestión construye un haz de relaciones entre dirección y cada sujeto que termina siendo desplazado de la realidad organizacional para focalizarse en su propia relación con el con la dirección.

Otra perspectiva diferente es la de Grinberg (2006). La gestión se vincula a la lógica del rendimiento y los resultados, pero trasciende a éstos. Los resultados no son sino el comienzo de un nuevo ciclo. Lo que importa son los procesos mediante los que se consiguen los logros. De este modo se construye una mirada permanente sobre la trayectoria de sujetos y unidades. Los sistemas de información se constituyen en dominios de saber que son interiorizados por los sujetos. Pero lo más importante de este modo de operar es que se construyen procedimientos para facilitar que los sujetos acepten responsabilidades. Este es el objetivo principal. La dirección promueve consensos que sirven de vehículo de presión a cada sujeto. De esta forma la gestión opera convirtiendo a todos en gestores. Todos terminan asumiendo su posición determinada por los indicadores y la trayectoria. De este modo la gestión, a partir de sus herramientas, corrige a los sujetos convirtiéndolos en empresarios de sí mismos.

El enfoque sobre la gestión de la sociología clínica (Gaulejac 2005), aporta algunos elementos fundamentales. Además de su análisis de la gestión como sistema de poder y control eficaz, subraya los efectos producidos en los sujetos transformados en recursos humanos para la producción. Lo característico de la gestión es el repertorio de presiones que produce a los sujetos obligados a internalizar su adhesión. En los guiones de carrera profesional no hay otra alternativa que ganar-ganar. El resultado es la aparición de malestares, acontecimientos críticos y efectos patológicos de gran alcance. El sistema managerial genera un modelo cultural en el que se exige la fusión con la empresa (Aubert y Gaulejac, 1993). Los discursos de la sociología clínica se adaptan a las transformaciones que se producen en el capitalismo cognitivo.

Los sistemas sanitarios son un campo de ensayo privilegiado de estas prácticas de la gestión. Los contratos-programa, las carteras de servicios, los sistemas de incentivos, los sistemas de información y la creciente evaluación. Todos ellos son consensuados con los profesionales, pero su sentido se proyecta en el largo plazo. Se supone que una tecnología tan individualizadora y eficaz puede debilitar la cohesión corporativa de los médicos. De esta forma se reconstituyen las bases de poder para los gestores y se modifica el campo organizativo a favor de éstos.

Desde distintas perspectivas se ha puesto de manifiesto la ineficacia de la gestión.en relación a sus objetivos. Se trata del penúltimo mito del capitalismo del final del siglo XX (Le Goff, 2000). Pero la mirada desde el paradigma de la gubernamentalidad, pone de manifiesto su eficacia en el disciplinamiento. Su gradual implementación modifica el medio organizativo sanitario y las bases del conflicto convencional entre administradores y profesionales, enunciado en la sociología médica. Los gerentes están dotados de recursos de conocimiento, proyecto y poder mucho más efectivos que los administradores burocratizados de antaño.

\section{LA PROFESIÓN MÉDICA EN LA REESTRUCTURACIÓN DE LOS SERVICIOS SANITARIOS}

El campo de la producción de los servicios sanitarios, y el sistema sanitario público en particular, registra los cambios analizados así como las tensiones que generan, que no siempre son manifiestas. Una de las consecuencias de la reestructuración del campo es la nueva posición de la profesión médica. Esta se encuentra entre las dos fuerzas emergentes: La industria biomédica y la 
gestión. En ambos casos, con referencia a épocas anteriores, la profesión médica se encuentra en desventaja. Tanto la industria como el gerencialismo, que representan las fuerzas que materializan el proyecto y los intereses fuertes que inspiran la mutación global, no tratan de desplazarla, sino de reducirla a la colaboración en las coordenadas de unos servicios de salud reformados. Esto significa una pérdida de autonomía profesional y una disminución de su poder en términos estratégicos. Pero este proceso de declive moderado, no es equivalente a los procesos de proletarización o desprofesionalización en los términos definidos por la sociología norteamericana de las profesiones (Guillén, 1990). La cuestión principal no radica en la complejización de las organizaciones de asistencia médica, que operan como conjuntos automatizados de los que se derivan procesos de división de trabajo multidimensionales gobernados por tecnoburocracias que configuran el trabajo médico, limitando severamente su autonomía.

El factor esencial de la reestructuración se deriva del papel asignado a la atención sanitaria en la nueva economía postfordista. El sistema público y la profesión médica son reconfigurados para adaptarse a los procesos productivos y sus modelos. El objetivo es la reforma del sistema público, desagregando sus redes asistenciales e introduciendo la competencia entre las mismas para adecuarlo al mercado. En el supuesto de que la reestructuración avance, las organizaciones públicas adoptarán progresivamente los modelos de las empresas aseguradoras privadas. El trabajo médico se puede homologar así con el estatuto de las múltiples profesiones que venden servicios a empresas en la economía postfordista. Se trata de trabajadores autónomos o semiautónomos regulados por una relación postsalarial con las empresas. En este contexto opera con facilidad la nueva gubernamentalidad. Los propios profesionales se autodisciplinan restringiendo las decisiones clínicas que conlleven recursos cuantiosos.

La tendencia a la progresiva desfuncionarización de los médicos del sector público implica la generación de procesos de estratificación en la profesión. La puesta en marcha de múltiples mecanismos de acreditación y evaluación, desarrollados por agencias externas, fomenta el principio de competencia, la individualización y el gobierno de la diferencia. Estas tecnologías de gobierno tienden a consolidar una diferencia sustantiva entre élites profesionales seleccionadas por sus vínculos con los proyectos de la industria y su posición preponderante en los mercados. Por debajo de un gradiente de posiciones se sitúan aquellos profesionales que tienen que subordinarse drásticamente a criterios eficientistas (Annandale, 1998).

En los últimos años se ha suscitado la cuestión del profesionalismo médico. Se entiende como un cuadro de valores consensuado que genera una norma interna y fomenta la cohesión en la profesión. Freidson (2001) ha codificado sus ventajas frente a otras formas de producción de servicios como la burocracia o el mercado. En las coordenadas del proceso de reestructuración profesional analizado hasta aquí, el profesionalismo tiene el riesgo de convertirse en una ideología profesional vaciada de contenido. La fragmentación de la profesión médica y su situación en el campo de fuerzas dominado por las empresas y los gestores, son factores adversos al desarrollo del profesionalismo.

Desde una perspectiva tan autorizada como la de Pardell (2003), se hace un inventario de las amenazas a la autonomía de la profesión médica derivada de los cambios sociales múltiples. Desde otras perspectivas, se subraya la relación entre el modelo de profesionalismo y la nueva gubernamentalidad (Fournier, 1999). La importación de los modelos empresariales para la producción de servicios sanitarios, pone de manifiesto la importancia de la cultura empresarial. El profesionalismo se entiende como un elemento que actúe a favor de la penetración de una cultura de servicios centrada en los clientes. El profesionalismo es el reverso de la cultura gerencial que pretende modificar las culturas profesionales médicas convencionales.

La industria biomédica, respaldada por su gran potencialidad productiva, toma la iniciativa decididamente, desarrollando un marketing intensivo en torno a sus proyectos y productos. La profesión comparece con el estatus de socio de la expansión terapéutica cuyo protagonismo se desplaza a la industria y sus productos. Los médicos son la vía de acceso principal a ellos. Pero la cuestión principal estriba en las definiciones. La comunicación de la industria erosiona la definición de los significados de la salud y 
la enfermedad, que hasta ahora correspondía a los médicos en exclusiva. La industria, que forma parte del conglomerado productivo, conecta mejor con las definiciones de bienestar imperantes y con el consumidor flexible que entiende su vida como un campo de experimentación del bienestar. Las empresas biomédicas tienen más facilidad para interactuar y transformar aspiraciones en necesidades.

La industria biomédica, convertida en una parte de la industria del bienestar, definido en términos de consumo, construye discursos y significaciones sobre la vida y la salud que tienen un impacto en el imaginario social. Como cualquier sector productivo en el presente, tiene que configurar su propia demanda a partir de las simbiosis entre las soluciones tecnológicas disponibles y las necesidades de los posibles clientes compradores. Este proceso productivo genera unos supuestos y sentidos que se interfieren con los producidos por la salud pública a partir del análisis epidemiológico y la etiología e impacto de las enfermedades.

La relación entre la industria y la profesión se ha complejizado. Los médicos son desposeídos de la exclusividad en cuestiones fundamentales, pero son considerados socios imprescindibles. Ellos facilitan el acceso a los pacientes necesarios para los ensayos clínicos. La consecuencia más importante de esta asociación es que introduce criterios de estratificación en el interior de la profesión médica. La articulación entre la investigación de la industria y la biomédica, con la preponderancia de la primera, introduce un criterio de jerarquización en la producción de conocimiento médico.

En el caso de la gestión el proceso es similar. La gestión es una tecnología de gobierno de las organizaciones muy poderosa. Las primeras generaciones de gerentes sanitarios se caracterizan por su débil profesionalización y su exceso de politización. Frente a los médicos parecen débiles representantes de un poder externo. Pero en términos estratégicos, el progreso de la gestión es incuestionable. La gestión opera produciendo consensos. En la elaboración de los contratos-programa, sistemas de información, carteras de servicios o de incentivos, participan activamente muchos cualificados profesionales. La fascinación ejercida sobre los mismos por los saberes empresariales es patente. Pero el consen- so obtenido se utiliza como fuerza de presión hacia cada profesional. Cada uno tiene que responder a sus evaluaciones y su trayectoria en el nuevo imperio del output. Los efectos disciplinarios de estas tecnologías políticas se hacen visibles en los últimos años. Disminuyen significativamente las actividades cooperativas y se genera una conformidad y uniformidad generalizada. Se debilita el tejido de actividades y organizaciones profesionales imperante con anterioridad (Irigoyen 2010).

La unidad y la cohesión de la industria contrasta con la alta segmentación y compartimentación de la profesión médica. Las sociedades de las distintas especialidades negocian con la administración sus propios intereses y en canales diferentes. Los vínculos horizontales entre especialidades son débiles, de modo que el conjunto de la profesión se encuentra desintegrado. La Federación de Asociaciones para la Defensa de la Sanidad Pública representa un foco de resistencia a la reestructuración entendida como colonización empresarial. En los últimos años aparecen algunas iniciativas críticas focalizadas en problemas específicos. La plataforma $\mathrm{No}$ Gracias (http://www.nogracias.eu ) constituye un indicio de activación de posiciones profesionales críticas en el campo sanitario.

La cuestión de fondo radica en el que la reestructuración de los servicios sirve al desarrollo de las fuerzas de mercado, consideradas como factor esencial del crecimiento económico. El sistema sanitario público, tal y como está configurado, es un obstáculo a este objetivo. La fragmentación de la provisión de servicios es una cuestión fundamental para el proyecto de reestructuración (Sánchez Bayle et al, 1996). El progreso de éste requiere segmentar los servicios en múltiples redes asistenciales relacionadas mediante vínculos de muy distinta naturaleza. Sólo de esta forma es posible la reducción de costos estableciendo niveles de asistencia de distintas calidades, como en el sistema de servicios personales que referencia esta transformación. El nuevo estado postfordista desempeña esta tarea central (Jessop, 1994). Los servicios sanitarios adquieren la condición de sector reformable para adaptarse al proceso de estructuración económica global.

El aspecto más relevante de estas reformas con respecto a la profesión médica radica en la 
eficiencia. Esta se define por la relación entre los costes y los resultados. El gasto farmacéutico está desbocado y en una sociedad de consumo avanzado es difícil frenarlo. El envejecimiento de la población, el aumento de enfermos crónicos y las dinámicas asistenciales determinan la multiplicación de pruebas, interconsultas, atención a episodios críticos y otras actividades de alto coste. Desde la racionalidad económica es necesaria la organización de un racionamiento, así como la transferencia al mercado de los sectores que puedan ser rentables. Los médicos son colaboradores imprescindibles en este proceso.

La crisis de costes representa la importación por parte del sistema público de la medicina gerenciada. Existen controversias acerca de sus resultados, pero tiende a priorizar la eficiencia sobre la calidad planteando conflictos éticos a numerosos profesionales (Conill, 1999). En un contexto organizativo de una empresa aseguradora el conflicto se resuelve mediante la fidelidad a la empresa. Pero en el sistema público en España, los médicos han dispuesto de abundantes medios y autonomía en sus decisiones. Las reformas en los últimos años comienzan a producir presiones de distinta naturaleza hacia la eficiencia. La interpretación realizada por los gestores de la medicina basada en la evidencia, en no pocas ocasiones tiene la pretensión de recortar la autonomía de las decisiones clínicas y convertir el trabajo médico en un conjunto de actos automatizados y que facilite las soluciones eficientes.

La medicina gerenciada es viable en contextos organizativos diseminados en múltiples servicios y contratos. Genera diversos problemas de acceso, de calidad de atención, de restricción de prestaciones y selección adversa penalizando los grupos con menos recursos y a los enfermos cuya asistencia necesita de más recursos (Ginzberg, 1999). La transformación de la atención sanitaria en un sector de consumo propicia elevados niveles resatisfacción con la escasa calidad asistencial (Millenson, 1997). La medicina gerenciada introduce un factor de desprofesionalización inquietante. Su generalización conlleva la creación de una superpoblación médica que facilite la flexibilidad del mercado de trabajo.

La atención médica, en proceso de reforma para adaptarse a los imperativos de los procesos de estructuración centrales, recepciona los modelos hegemónicos de producción y consu- mo: La economía del High tech-low cost. La aplicación de este modelo en el la atención sanitaria genera interrogantes de gran calado acerca de su pertinencia y su viabilidad, así como sobre sus impactos en los sectores de población en desventaja social. La atención médica no puede asumir el supuesto de crecimiento permanente que inspira al sistema productivo del presente. Repensar los problemas de la asistencia sanitaria desde otras perspectivas vuelve a ser una cuestión fundamental. Las sombras de Illich (1975), Skrabanek (1999) y otras voces críticas, reaparecen en el curso de la reestructuración.

La reestructuración de la profesión médica reformula el concepto convencional de medicalización. La nueva medicalización implica la preponderancia de la industria y las empresas que la desarrollan mediante la explotación intensiva de herramientas mediáticas derivadas del repertorio del penúltimo marketing. Los médicos son acompañantes de la puesta en escena de la magia comunicacional mediática asociada al capitalismo cognitivo. La explosión de la medicina seductora es el ingrediente principal de la medicalización en el presente. Esta se expande por la videoesfera articulada a la industria del cuerpo.

Atrapada por dos fuerzas emergentes de la época, focalizada en la producción de servicios de calidad técnica, la profesión médica se toma distancia respecto a la salud colectiva y sus determinantes (Alvarez, García-Gil y Solano, 2008). El progreso de los medios diagnósticos y terapéuticos, junto con la mejora de los niveles de vida, no disminuyen la demanda ni la importancia de los problemas de salud colectiva. El reverso de la sociedad tecnológica y de consumo es la existencia de agudas desigualdades y lados oscuros del progreso, que se materializan en complejas problemáticas de bienestar y salud. La concurrencia de la profesión médica con la Epidemiología, la Salud Pública o las Ciencias Sociales parece imperativa desde esta perspectiva. Pero su campo relacional restringido a las fuerzas hegemónicas que impulsan la reestructuración, determina la generalización de una cosmovisión descentrada respecto a la complejidad de las realidades sociales y las problemáticas de la salud colectiva. La profesión médica se encuentra extraviada en el laberinto tecnológico, comercial y del consumo desbocado. 


\section{BIBLIOGRAFÍA}

ACEBILlo, J. y ARTELls, J.J. (2004) La biomedicina como factor de creación de valor y crecimiento económico. Revista de Administración Sanitaria 2 (3). 357-373.

Alvarez-Girón, M., García-Gil, C. y Solano, A.,(2008). Los determinantes de salud de las poblaciones y de las desigualdades sociales en salud. En Escolar, A. (ed) Primer Informe sobre Desigualdades y Salud en Andalucía. Cádiz: Asociación para la defensa de la Sanidad Pública de Andalucía.

Annandale, E. (1998) The Sociology of Health and Medicine. Cambridge: Polity Press.

Angell, M. (2004). The Ttruth About the Drug Companies: How They Deceive Us and what to Do About it. Nueva York: Random House.

Aubert, N. y V. De Gaulejac. (1993) El coste de la excelencia. Barcelona: Paidós.

Ayestarán, I. (2007). Capitalismo cognitivo en la economía high tech y low cost: de la ética hacker a la wikinomía. Argumentos de Razón Técnica 10. 89-123.

BALL, S.J. (1993) La gestión como tecnología moral. Un análisis ludista. En Ball, S.J. (comp.) Foucault y la educación. Disciplinas y saber Madrid: Morata 155-168.

BERARDI, F. (2003) La fábrica de la infelicidad. Nuevas formas de trabajo y movimiento global. Madrid: Traficantes de Sueños.

Blech, J. (2005) Los inventores de enfermedades. Cómo nos convierten en pacientes. Barcelona: Destino.

BORRACCI, R. (2001) De la medicina basada en la evidencia a la medicina basada en la seducción. Revista Fundación Facultad de Medicina.11. 20-23.

- (2006) La medicina basada en la seducción y la comunicación al público de temas médicos. Revista Argentina de Cardiología Vol. 74 (4).

BRUCNER, P. (1996). La tentación de la inocencia. Barcelona: Anagrama.

Conill, J. (1999) ¿Adiós a Hipócrates? Conflictos éticos de la medicina gestionada. Claves de Razón Práctica $98.49-54$

Costa, J. (1999). La comunicación en acción. Informe sobre la nueva cultura de la gestión. Barcelona: Paidós.

DRUCKER, P. (1993). La sociedad poscapitalista. Barcelona: Apóstrofe.

Foucault, M. (1981) La gubernamentalidad, en Castel, R. et al. Espacios de poder. Madrid: La Piqueta 9-26.

FourNiER, V. (1999) The appeal to professionalism as a disciplinary mechanism. Social Review 47 (2). 280307.

Freidson, E. (2001) Professionalism. The Third Logic. Cambridge: Polity Press.

GAULEJAC, V. de (2005) La société malade de la gestion. Ideologie gestionnaire, pouvoir managérial et harcèlement social. París: Éditions du Seuil.

GAY P. DU (2000) Entrepreneurial Governance and Public Management: The Anti-Bureaucrats. En J. Clarke, Sh Gerwirtz and E. Mclaughlin (eds). New Managerialism, new Welfare? Londres: Sage.

GinZBERG, E. (1999).The uncertain future of Managed Care. New England Journal of Medicine. 340. 144-146.

GRINBERG, S. (2006) Educación y gubernamentalidad en las sociedades de gerenciamiento. Revista Argentina de Sociología año Vol.4 (6) 67-87.

GuILLÉN, M. (1990) Profesionales y burocracia: Desprofesionalización, proletarización y poder profesional en las organizaciondes complejas REIS 51.35-51

HASSARD, J. and M. PARKeR (eds) (1997) Postmodernism and organizations Londres: Sage.

ILlich, I. (1975) Némesis médica. La expropiación de la salud Barcelona: Barral.

IRIGOYEN, J. (2007) La reinvención de los pacientes. El cliente siempre tiene razón. Salud 2000 113.20-25.

- (2010) El declive de los discursos comunitarios en la reestructuración de la Atención Primaria en España. Capítulo 10 en Palomo, L. (Coord) Expectativas y realidades en la Atención Primaria española. Madrid: Fundación $1^{\circ}$ de mayo EdicionesGPS

JARA, M. (2007). Traficantes de salud .Barcelona: Icaria \& Antrazyt.

- (2009) La salud que viene. Nuevas enfermedades y el marketing del miedo. Barcelona: Península.

Jessop, B. (1994) Postfordism an the state. En A. Amin (ed) Postfordism: A Reader. Oxford: Blackwell.

LAZZARATO, M. El ciclo de la producción inmaterial. http://enmedio.info//el-ciclo-de-la-producción-inmaterial/ Consultado 26/06/2010 
Lazzarato, M. y A. Negri. (2001). Trabajo inmaterial, formas de vida y producción de la subjetividad. Río de Janeiro: DP\&A Editora.

Le Goff, J-P (2000). Les illussions du Management. París: La Découverte.

McKinlay, A., Starkey, K. (Eds) (1998). Foucault, Management and Organization Theory: From Panopticom to Technologies of self. Londres: Sage Publications.

MARAzZI, C. (2003). El sitio de los calcetines. El giro lingüístico de la economía y sus efectos sobre la politica. Madrid: Akal.

Millenson, M. L. (1997). Demanding Medical Excellence. Chicago: University Chicago Press.

MOULIER- BoutANG, Y. (2007). Le capitalisme cognitif:Le nouvelle grande transformation .París: Ed. Amsterdam

OMinAmi, C. (Ed.) (1986). La tercera revolución industrial. Impactos internacionales del actual viraje tecnológico. Buenos Aires: Grupo Editor Latinoamericano.

Pardell, H. (2003) ¿Tiene sentido hablar de profesionalismo hoy? Educación Médica 6 (2). 63-80.

PARKer, M (2002) Against Management: Organization in the Age of Managerialism. Cambridge: Polity Press.

PignarRe, P. (2005). El gran secreto de la industria farmacéutica. Barcelona: Gedisa

QuintanA, F. (2009). Actividad sociocognitiva y contextos postfordistas. Revista Internacional de Sociología. Vol.67 n². 347-371.

Rose. N. (1997) El gobierno en las democracias liberales avanzadas: del liberalismo al neoliberalismo. Archipiélago 29. 25-40.

- (2007) ¿La muerte de lo social? Reconfiguración del territorio de gobierno. Revista Argentina de Sociología Vol 5 (8) 111-150.

Rose, N. y Miller, P (1991) Political power Beyond the State: Problematics of Government. British Journal of Sociology 43 (2) 172-205.

Sánchez Bayle (ed) (1996) El sistema sanitario en España. Evolución, situación actual, problemas y perspectivas. Madrid: Los Libros de la Catarata.

Skrabanek, P. (1999) La muerte de la medicina con rostro humano. Madrid: Díaz de Santos

Sobrino,J.A (2010) El complejo médico-industrial y los análisis clínicos. Dempeus per la Salud Pública. http://dempeus.nireblog.com/post/2010/04/el-complejo-medico-industrial-y-los-ensayos-clinicos 04/05/2010 